\title{
Hemoperfusion as a Potential Treatment for Critically III COVID-19 Patients with Cytokine Storm
}

\author{
Kamran Shadvar $^{\mathrm{a}}$ Ali Tagizadiyeh ${ }^{\mathrm{b}}$ Ali Akbar Gamari ${ }^{\mathrm{a}}$ Hassan Soleimanpour $^{\mathrm{c}}$ \\ Ata Mahmoodpoor ${ }^{a}$ \\ aDepartment of Anesthesiology, Faculty of Medicine, Tabriz University of Medical Sciences, Tabriz, Iran; \\ bTuberculosis and Lung Research Center, Tabriz University of Medical Sciences, Tabriz, Iran; 'Department of \\ Emergency Medicine, Medicine, Faculty of Medicine, Tabriz University of Medical sciences, Tabriz, Iran
}

After COVID-19 outbreak in China in late 2019 and change to pandemic in early 2020 , the disease became a clinical threat to the whole world [1]. Urgent clinical guidance for intensive management of critically ill patients is needed. To date, therapeutic options for severe COVID-19 remain limited, and this disease has high mortality in patients admitted to intensive care units (ICUs) [2]. Huang et al. [5] reported the clinical features and cytokine profile of critically ill patients with COVID-19 in Wuhan, China, and suggested that a cytokine storm had a high mortality rate and was a leading cause of organ dysfunction and death in these patients. Infectious and noninfectious triggers can result in a cytokine storm, progressing to vasoplegic shock and finally multiorgan dysfunction syndrome (MODS) [3]. Early detection and appropriate management of this storm can be an important intervention in decreasing the mortality of critically ill COVID-19 patients. From the first admission of critically ill COVID-19 patients in our ICU on March 10,2020 , we had 8 cases of hemoperfusion in our patients. Our standard treatment protocol beside antiviral therapy for these patients included using $\mathrm{HFNC/noninvasive}$ ventilation (Helmet) based on availability as the first choice in patients with acute respiratory failure. If pa- tients failed these interventions and were awake and cooperative, we would try early application of prone positioning (cooperative prone positioning) with noninvasive ventilation/HFNC as long as they tolerate. If the situation went wrong, we would try intubation and mechanical ventilation with lung protective strategy (tidal volume of 4-6 mL/predicted body weight and adjustment of it based on driving pressure of $14 \mathrm{~cm} \mathrm{H}_{2} \mathrm{O}$ and plateau pressure of $30 \mathrm{~cm} \mathrm{H}_{2} \mathrm{O}$ ). PEEP was adjusted and titrated based on stress index and driving pressure. However, if increasing PEEP resulted in improved oxygenation, we would increase the PEEP number, but if increasing PEEP did not result in improving oxygenation, we would consider the patient as a nonresponder and would not increase PEEP to more than $12 \mathrm{~cm} \mathrm{H}_{2} \mathrm{O}$. We used adjunctive therapies in patients with cytokine storm without considering the method of ventilation. If the patient had at least 2 of the criteria in Figure 1, the situation would be considered as cytokine storm. Cytokine storm was diagnosed in 8 patients; 4 of them were nonintubated. One patient received 4 sessions of hemoperfusion, and 1 patient received just 2 sessions as he died before the third session. The others received 3 sessions with Jafron hemoabsorber (HA380 Disposable Hemoperfusion Cartridge). Hemoperfusion

$\begin{aligned} & \text { karger@karger.com } \\ & \text { www.karger.com/bpu }\end{aligned}$
Karger ${ }^{\prime /}$

Ata Mahmoodpoor

Anesthesiology and Critical Care, Tabriz University of Medical Sciences Daneshkade Street

Tabriz 5157663314 (Iran)

amahmoodpoor@yahoo.com 


\begin{tabular}{|l|}
\hline \\
Lymphopenia $<0.8$ \\
Lactate dehydrogenase $>250 \mathrm{U} / \mathrm{L}$ \\
Ferritin $>600 \mathrm{mg} / \mathrm{mL}$ \\
C reactive protein $>120 \mathrm{mg} / \mathrm{L}$ \\
D-dimer $>1,000 \mathrm{mg} / \mathrm{mL}$ \\
H-score $>200$ \\
Acute development of organ dysfunction \\
(high SOFA score)
\end{tabular}

Fig. 1. Criteria considered as severity in critically ill patients with COVID-19.

was combined with renal replacement therapy in 2 patients, but it was exclusively performed in the remaining 6 patients. Three of our patients died and others survived. One of the expired patients had AKIN stage 3 and underwent hemoperfusion with CRRT, and the other 2 patients did not develop AKI during their ICU stay. The demographic baseline characteristics of patients and clinical parameters before and after hemoperfusion are shown in Table 1. Informed consent was taken from all patients or their next of kin to publish their case. The "cytokine storm" in patients with new coronavirus is an important cause of death in critically ill patients. According to the Sixth Edition of Diagnosis and Treatment Scheme for Novel Coronavirus Pneumonia, the use of hemoperfusion, blood/plasma filtration, and other extracorporeal blood purification techniques may be considered for critically ill patients with high inflammatory response [4]. The understanding of the cytokine storm mechanism(s) and its profile are crucial to developing effective therapeutic interventions in COVID-19. So, for early diagnosis and treatment of this state, we need to identify a biomarker panel before the administration of selective immunosuppressive therapy [5]. Among key cytokines, IL-6 has attracted high levels of interest, and antibodies that block the IL-6 receptor (tocilizumab) are currently under phase $2 / 3$ clinical trials for the potential treatment of COVID-19 [6]. However, as we did not have possibilities for real-time assessment of IL-6, we did not use tocilizumab in our center. In organ dysfunction syndromes, when pharmacological therapy is not available or efficacious, mechanical ventilation and hemodynamic support seem to be the only possible therapeutic and supportive strategy [7]. In this situation, extracorporeal therapies such as
Table 1. Demographic characteristics of patients in this study

\begin{tabular}{|c|c|c|}
\hline Variable & Value & \\
\hline Age, years & $44.25 \pm 12.56$ & \\
\hline Weight, kg & $76.35 \pm 14.35$ & \\
\hline Sex, M/F & $4 / 4$ & \\
\hline \multicolumn{3}{|l|}{ Comorbidities } \\
\hline Diabetes mellitus & 2 & \\
\hline Cardiovascular disease & 3 & \\
\hline Kidney dysfunction & 1 & \\
\hline Hyperlipidemia & 1 & \\
\hline Smoking & 2 & \\
\hline Neurologic dysfunction & 1 & \\
\hline $\mathrm{APACHE}$ & $24(17-32)$ & \\
\hline SOFA & $13(9-17)$ & \\
\hline MV duration, days & $12.50 \pm 5.30$ & \\
\hline NIV duration, days & $7.30 \pm 3.45$ & \\
\hline \multirow[t]{2}{*}{ ICU LOS, days } & $15.40 \pm 4.50$ & \\
\hline & $\mathrm{BBP}$ & $\mathrm{ABP}$ \\
\hline Ferritin & $667.59 \pm 91.36$ & $532.14 \pm 51.78$ \\
\hline D-dimer & $1,205.43 \pm 49.07$ & $843.55 \pm 129.06$ \\
\hline CRP & $137.25 \pm 49.07$ & $86.11 \pm 18.35$ \\
\hline H-score & $265.38 \pm 43.67$ & $158.36 \pm 41.59$ \\
\hline Lactate & $3.21 \pm 1.47$ & $2.30 \pm 1.56$ \\
\hline Cardiac index & $2.44 \pm 0.64$ & $2.68 \pm 0.34$ \\
\hline Capillary refill time(s) & $3.21 \pm 0.59$ & $2.75 \pm 0.75$ \\
\hline Procalcitonin, $\mathrm{ng} / \mathrm{mL}$ & $2.45 \pm 0.37$ & $2.13 \pm 0.45$ \\
\hline $\mathrm{PaO}_{2} / \mathrm{FiO}_{2}$ & $110.35 \pm 23.42$ & $143.24 \pm 18.37$ \\
\hline Plateau pressure, $\mathrm{cm} \mathrm{H}_{2} \mathrm{O}$ & $28.05 \pm 2.14$ & $26.54 \pm 3.75$ \\
\hline Driving pressure, $\mathrm{cm} \mathrm{H}_{2} \mathrm{O}$ & $15.04 \pm 2,065$ & $12.56 \pm 3.21$ \\
\hline Lung compliance & $34.25 \pm 9.10$ & $40.36 \pm 5.44$ \\
\hline Airway resistance & $11.05 \pm 3.46$ & $10.56 \pm 2.84$ \\
\hline EVLW, mL/kg & $365.45 \pm 3.65$ & $289.36 \pm 39.43$ \\
\hline
\end{tabular}

ABP, after blood purification; APACHE, Acute Physiology and Chronic Health Evaluation; BPB, before blood purification; ICU LOS, intensive care unit length of stay; CRP, C-reactive protein; EVLW, extravascular lung water, measured by PICCO (transpulmonary thermodilution); M/F, male/female; MV, mechanical ventilation; NIV, noninvasive ventilation.

hemofiltration or hemoperfusion offer a new possibility to support different organs in a multiple organ dysfunction condition. There are some reports about the significant benefit from hemoperfusion with the use of direct hemoperfusion with cartridges containing highly biocompatible sorbents and microporous resins in a large proportion of patients with complicated COVID-19 syndromes [8]. The mechanism is nonspecific removal of the peaks of the circulating cytokines in both the pro- and anti-inflammatory sides, which results in the reduction of circulating levels of cytokines and may allow the immune 
system of the patient to redirect the immunocompetent cells to the source or site of inflammation [9]. After the first application of HA380 hemoperfusion, our patients showed an improvement in pulmonary markers, dynamic indices, and LDH level was decreased. A decrease in CRP, ferritin, procalcitonin, cardiac index, and extravascular lung water levels was evident following the second application. Ferritin level and extravascular lung water declined after the third application. Overall, improved levels of inflammatory, respiratory, and perfusion parameters were evident following the application of HA380 hemoperfusion 6-8 h daily for 3 consecutive days; however, no effect was evident on the prognosis. We performed hemoperfusion after the diagnosis of cytokine storm during first $48 \mathrm{~h}$ of ICU admission. We used incremental dosage of anticoagulation both to maintain circuit patency and to manage the thrombophilia of the patient. We started low molecular heparin $10 \mathrm{IU} / \mathrm{kg} / \mathrm{h}$, but in some patients a higher dosage of up to $20 \mathrm{IU} / \mathrm{kg} / \mathrm{h}$ may be required to ensure circuit patency. Regarding COVID-19 patients, extracorporeal therapies represent the perfect combination of organ support, and endotoxin and cytokine removal to provide blood purification in these patients. These therapies can be considered as therapeutic approach for the prevention of organ dysfunction due to cytokine storm associated with severe COVID-19.

Finally, our results showed that hemoperfusion can decrease the level of inflammation and organ dysfunction in critically ill patients with COVID-19. We need more studies to show the best time for implementation of hemoperfusion and number of its sessions in outcome of these patients. Moreover, all ICU staff and physicians should be familiar with the concept of hemoperfusion in management of critically ill patients. So, hemoperfusion (early application) combined with appropriate antiviral therapies and supportive therapy may be considered as an adjunctive treatment for critically ill COVID-19 patients. For the moment, the pathophysiological rationale is the only reason to recommend that application of hemoperfusion, and a personalized evaluation is advised. In addition, if such intervention is being considered, it seems logical to apply it as soon as possible.

\section{Acknowledgements}

We want to acknowledge all general ICU staff of Imam Reza Hospital, and Masoud Hamidi, Mojtaba Mohammadzadeh, and Roqhayyeh Asghari who helped us in management of these patients and conduction of the study.

\section{Conflict of Interest Statement}

The authors have no conflicts of interest to declare.

\section{Funding Sources}

This study was funded by research deputy of Tabriz University of Medical Sciences.

\section{Author Contributions}

A.M.: hypothesis of study, literature review, initial drafting, and acquisition of the data. A.T.: hypothesis of study and literature review. K.Sh.: data collection, literature review, and critical revision. A.Q.: data collection and critical revision. H.S.: data collection, literature review, and initial drafting. All authors read and approved the final version of the manuscript.

\section{References}

1 Murthy S, Gomensall C, Fowler RA. Care for critically ill patients with COVID-19. JAMA. 2020;323(15):1499-1500

$2 \mathrm{Wu}$ Z, McGoogan JM. Characteristics of and important lessons from the coronavirus disease 2019 (COVID-19) outbreak in China: summary of a report of 72314 cases from the chinese center for disease control and prevention. JAMA. 2020;323(13):1239-42.

3 Huang C, Wang Y, Li X, Ren L, Zhao J, Hu Y, et al. Clinical features of patients infected with 2019 novel coronavirus in Wuhan, China. Lancet. 2020;395(10223):497-506.
4 Diagnosis and Treatment Protocol for Novel Coronavirus Pneumonia. (Trial Version 6, Revised). Available from: wwwkankyokansen org,files`jsipcsprotocolV65.

5 Gong J, Dong H, XiaS Q, Huang YZ, Wang D, Zhao Y, et al. Correlation analysis between disease severity and inflammationrelated parameters in patients with COVID-19 pneumonia. medRxiv. 2020.

6 Zhang C, Wu Z, Li J-W, Zhao H, Wang G-Q. The cytokine release syndrome (CRS) of severe COVID-19 and Interleukin-6 receptor (IL-6R) antagonist Tocilizumab may be the key to reduce the mortality. Int J Antimicrob Agents. 2020;55(5):105954.
7 Bouadma L, Lescure FX, Lucet JC, Yazdanpanah Y, Timsit JF. Severe SARSCoV-2 infections: practical considerations and management strategy for intensivists. Intensive Care Med. 2020;46(4):579-82.

8 Ankawi G, Fan W, Pomarè Montin D, Lorenzin A, Neri M, Caprara C, et al. A new series of sorbent devices for multiple clinical purposes: current evidence and future directions. Blood Purif. 2019;47(1-3):94-100.

9 Rimmelé T, Kellum JA. Clinical review: blood purification for sepsis. Crit Care. 2011;15(1): 205 